OPEN ACCESS

Edited by:

Sandro Massao Hirabara,

Universidade Cruzeiro do Sul, Brazil

Reviewed by:

Roberto Bazotte,

State University of Maringá, Brazil

Xin Cui,

Georgia State University,

United States

${ }^{*}$ Correspondence:

Yanchun $\mathrm{Hu}$

hychun114@163.com

${ }^{\dagger}$ These authors have contributed equally to this work and share first authorship

Specialty section: This article was submitted to Nutritional Immunology, a section of the journal

Frontiers in Immunology

Received: 16 December 2021 Accepted: 26 January 2022

Published: 15 February 2022

Citation:

Ren Z, Okyere SK, Xie L,

Wen J, Wang J, Chen Z, Ni X, Deng J and Hu Y (2022) Oral Administration of Bacillus toyonensis

Strain SAU-20 Improves Insulin Resistance and Ameliorates Hepatic Steatosis in Type 2 Diabetic Mice.

Front. Immunol. 13:837237. doi: 10.3389/fimmu.2022.837237

\section{Oral Administration of Bacillus toyonensis Strain SAU-20 Improves Insulin Resistance and Ameliorates Hepatic Steatosis in Type 2 Diabetic Mice}

\author{
Zhihua Ren ${ }^{1,2 \dagger}$, Samuel Kumi Okyere ${ }^{1,2 \dagger}$, Lei Xie ${ }^{1,2}$, Juan Wen ${ }^{1,2}$, Jiayi Wang ${ }^{1,2}$, \\ Zhengli Chen ${ }^{1,2}$, Xueqin $\mathrm{Ni}^{1,2}$, Junliang Deng ${ }^{1,2}$ and Yanchun $\mathrm{Hu}^{1,2,3^{*}}$ \\ 1 Key Laboratory of Animal Disease and Human Health of Sichuan Province, Sichuan Agricultural University, Chengdu, China \\ ${ }^{2}$ Key Laboratory of Animal Diseases and Environmental Hazards of Sichuan Province, College of Veterinary Medicine, \\ Sichuan Agricultural University, Chengdu, China, ${ }^{3}$ New Ruipeng Pet Healthcare Group Co., Ltd. Shenzhen, China
}

In this study, the ameliorative effects of Bacillus toyonensis-SAU-20 (B. toyo SAU-20), a new probiotic strain isolated and identified by our laboratory from Ageratina adenophora, on the development of insulin resistance and hepatic steatosis in type 2 diabetic (T2DM) mice was investigated. Thirty Specific-pathogen free Kunming (SPFKM) mice were randomly allocated to three groups: control, high fat diet/streptozotocin (HFD/STZ), and $\mathrm{HFD} / \mathrm{STZ}+B$. toyo SAU-20 groups with oral administration of $B$. toyo SAU-20 for 35 days. Biochemistry parameters, glucose tolerance, and insulin resistance were measured in the blood whereas histological analysis, inflammatory cytokines and lipogenic genes in the liver tissues. The results showed that, the levels of serum glucose, lipid profile, mRNA expression of lipogenic related genes and pro-inflammatory cytokines were significantly increased in T2DM mice. However, after B. toyo SAU-20 administration, the elevation of these parameters was significantly suppressed $(P<0.05)$. In addition, the feeding of $B$. toyo SAU-20 significantly improved the morphological changes of the liver with significant alleviation of dyslipidemia, oxidative stress status and inflammation $(P<0.05)$ indicating the ameliorating effect of $B$. toyo SAU-20 in hepatic steatosis in T2DM. Therefore, we concluded that, B. toyo SAU-20 alleviated insulin resistance and hepatic steatosis by improving the lipid profiles, antioxidant status and downregulating lipogenic genes as well as pro-inflammation cytokines expression.

Keywords: type 2 diabetes, hepatic steatosis, Bacillus toyonensis SAU-20, lipogenic genes, insulin resistance

\section{INTRODUCTION}

Type 2 diabetes mellitus (T2DM) is a metabolic disorder that possess threat to human and animals (1). Reports have shown that by 2030 the world diabetes population would reach 439 million (2). In addition, developing countries are highly prone to this metabolic disorder in the near future according to the World Health Organization (3). People suffering from T2DM have higher risk 
(35-80\%) of developing hepatic steatosis compared to diabetic free individuals (4). Insulin resistance is highly associated with metabolic syndrome, non-alcoholic fatty liver (NAFLD) (5). The liver is associated with the maintenance of lipid and energy homeostasis, and storage of excessive lipids in the liver as fat droplets, which when accumulated results in oxidative stress, inflammation, insulin resistance and diabetes (6). Furthermore, another key metabolic concern of diabetes is the unusual deposition of triglycerides in hepatocytes, which further stimulates hepatic steatosis (7).

B. toyo SAU-20 isolated from Ageratina adenophora an invasive plant (8-10) has showed good functional properties such as improving growth performance, antioxidant capacity, and gut integrity (11), of which are evident for the potential beneficial activity of $B$. toyo SAU-20 in vivo. This study was performed to examine the effect of $B$. toyo SAU-20 on fatty acid profile and liver functions in the HFD/STZ-induced T2DM in mice in order to reveal the treatment effects of probiotics in liver disorders such as NAFLD and its potential mechanisms, such as insulin resistance, hyperglycemia, oxidative stress, and inflammation.

\section{MATERIAL AND METHODS}

\section{Sample Collection}

Culture media were purchase from Qingdao Hope BioTechnology Co., Ltd., Qingdao, China and Streptozotocin (STZ) was purchased from Solarbio solabao Beijing solabao Technology Co., Ltd, China. Mice, basal and high fat diet (Table 1) were purchased at the Chengdu Dashuo Experiment Animal Co. Ltd. Chengdu, China. B. toyo SAU-20 (Accessory No. MW287199) was obtained from the College of Veterinary Medicine (Professor Yanchun Hu's lab), Sichuan Agricultural University, China.

\section{Preparation of Probiotic Bacteria Suspensions}

B. toyo SAU-20 were prepared by culturing in LB broth and incubating anaerobically at $37^{\circ} \mathrm{C}$ for $72 \mathrm{~h}$. The bacterial cells were separated by centrifugation $(3500 \mathrm{~g}, 5 \mathrm{~min}$ ) to remove the LB broth, and washed twice in $0.85 \% \mathrm{NaCl}$ (Sigma), and then resuspended in $0.85 \% \mathrm{NaCl}$ to a final concentration of $10^{7}$ $\mathrm{CFU} / \mathrm{mL}$ and stored at $4^{\circ} \mathrm{C}$.

TABLE 1 | Feed composition.

\begin{tabular}{lclc}
\hline $\begin{array}{l}\text { Normal diet } \\
\text { Ingredients }\end{array}$ & Content $\mathbf{g} \mathbf{k g}$ & $\begin{array}{l}\text { High fat diet } \\
\text { Ingredients }\end{array}$ & Content $\mathbf{g} \mathbf{k g}$ \\
\hline Water & 94 & Water & 93 \\
Carbohydrate & 675 & Carbohydrate & 342 \\
Protein & 190 & Protein & 134 \\
Fat & 51 & Fat & 143 \\
Fiber & 36 & Fiber & 27 \\
Ash & 62 & Ash & 44 \\
Calcium & 11.3 & Calcium & 8.3 \\
Phosphorus & 8.6 & Phosphorus & 7.1
\end{tabular}

\section{Experimental Animal and Design}

Thirty (30) Specific-pathogen free Kunming (SPFKM) male mice (5 weeks old; BW 25-30 g) were purchased from the Chengdu Dashuo Experiment Animal Co. Ltd. Chengdu, China. The animals were kept in plastic cages in an animal house maintained with a constant temperature $\left(22 \pm 2^{\circ} \mathrm{C}\right)$ and humidity $(65 \pm 5 \%)$ under a 12 -h light/12-h dark cycle. Mice were fed and given water $a d$ lib. This study was approved by the Institutional Animal Care and Use Committee of Sichuan Agricultural University, Sichuan, China, under the permit number DKY-B2019603005.

The mice diabetes model was established by the feeding of high-fat diet (HFD) for 6 weeks and the intraperitoneal injection of streptozotocin (STZ) solution (dissolved in a $0.01 \mathrm{M}$ citrate buffer, pH 4.5; Solarbio Science and Technology Co. Ltd., Beijing, China) with the dose of $35 \mathrm{mg} / \mathrm{kg}$ body weight for 3 consecutive days on the last day of the $6^{\text {th }}$ week (12). $72 \mathrm{~h}$ after the injection, fasting blood glucose (FBG) was measured using a blood glucose meter (Bayer). The diabetes model was recognized as successfully established when the blood glucose level was $>11.1 \mathrm{mmoL} / \mathrm{L}$ (13). Mice injected with equal amounts of precooling citrate buffer solution, $\mathrm{pH} 4.5$ were used as controls $(\mathrm{n}=10)$. Immediately, the model mice were randomly divided into a Diabetic group (DBG) $(\mathrm{n}=10)$ that was fed HFD $+1 \mathrm{~mL} 0.9 \%$ normal saline daily oral gavage and Diabetic $+B$. toyo SAU-20 group $(\mathrm{DBG}+B$. toyo SAU-20) $(\mathrm{n}=10)$ that was fed HFD $+1 \mathrm{~mL}$ of $10^{7} \mathrm{CFU} / \mathrm{mL} B$. toyo SAU-20 oral gavage aside the control group $(\mathrm{C})(\mathrm{n}=10)$ fed basal diet and $1 \mathrm{ml}$ of $0.9 \%$ normal saline oral gavage for 5 weeks starting from the day of allocation of mice to their various groups. Feed and water intake was monitored and recorded daily throughout the experimental period. Feed and clean water was provided ad libitum. Water bottles were washed every week and fresh drinking water was placed in it for the next week's administration. The bedding material (wood shavings) was also changed weekly. To administer the B. toyo SAU-20, new stocks were generated each week in LB and their viability was monitored by serial dilution and viable cell count using LB agar respectively.

\section{Oral Glucose Tolerance Test}

At the last day of the last week of the administration of $B$. toyo SAU-20, oral glucose tolerance test (OGTT) was performed. Mice were fasted for $12 \mathrm{~h}$ and blood glucose values were determined (time $=0 \mathrm{~min}$ ). Then mice were orally administered glucose $(1 \mathrm{~g} / \mathrm{kg} \mathrm{BW})$ and blood glucose levels were measured at 30, 60, 90 and $120 \mathrm{~min}$ from the tail vein using the glucose meter. Blood was collected from the tail vein using the syringe and few drops $(2-5 \mu \mathrm{L})$ of the blood was immediately poured on the test strips of the glucose meter for the reading of glucose level in the blood. The sensitivity of the test strip was $0.1 \mathrm{mmol} / \mathrm{L}$.

\section{Blood and Tissue Sample Collection}

At the end of the experiment (week 12), mice were fasted for $12 \mathrm{~h}$ and were anesthetized in an anesthesia chamber filled with $2.5 \%$ sevoflurane of $\mathrm{O}_{2}$ at a flow rate of $0.9-1 \mathrm{~L} / \mathrm{min}$ for up to $6 \mathrm{hr}$ (14). Blood samples were collected from the inferior vena cava and 
centrifuged at $4000 \times \mathrm{g}$ for $10 \mathrm{~min}$ at $4^{\circ} \mathrm{C}$, and the separated serum was stored at $-80^{\circ} \mathrm{C}$ for further assays. Tissue samples (liver) were immediately removed, rinsed, and stored at $-80^{\circ} \mathrm{C}$ or fixed in $10 \%$ paraformaldehyde solution.

\section{Biochemical Parameters}

Glycogen and insulin were measured using ELISA kits (Jiangsu Jingmei Biological Technology Company Limited, Jiangsu, China). Lipid profiles, including total cholesterol (TC), total triglyceride (TG), LDL-cholesterol (LDL-C), and HDL cholesterol (HDL-C) were measured by commercial kits (Jiangsu Jingmei Biological Technology Company Limited, Jiangsu, China). Homeostatic model assessment of insulin resistance (HOMA-IR), used to quantify insulin resistance, was calculated as: HOMA-IR = Fasting blood glucose $(\mathrm{mmol} / \mathrm{L}) \times$ Fasting blood insulin $(\mathrm{mU} / \mathrm{L}) / 22.5(15,16)$. The levels of glycogen, glutathione (GSH), and malondialdehyde (MDA), and the activities of superoxide dismutase (SOD) in mice serum and livers were determined using commercial kits from Jiangsu Jingmei Biological Technology Company Limited, Jiangsu, China.

\section{Liver Histological Analysis}

Livers were fixed into $4 \%$ parafomaldehyde. Then the tissues were embedded in paraffin and sectioned for $5 \mu \mathrm{m}$ thick. Hematoxylinensin (H\&E) staining was used for liver pathological evaluation. $\mathrm{H} \& \mathrm{E}$ staining kits were obtained from Jiancheng Bioengineering Institute (Nanjing, China). All kits were used according to the corresponding manufacturers' instructions Liver steatosis score was numerically recorded following the method of Qayyum et al. (17).

\section{Enzyme-Linked Immunosorbent Assay}

Liver tissues were washed with PBS, $0.1 \mathrm{~g}$ of the sample tissue was weighed and homogenized with $0.9 \mathrm{~mL}$ ice-cold PBS in a glass homogenizer, and then the mixture was centrifuged $(3000 \mathrm{rpm}$, $20 \mathrm{~min}$ ) to obtain the supernatant. Furthermore, we determined the protein concentration in the supernatant using a Total Protein Assay kit (Nanjing Jiancheng Bioengineering Institute, Nanjing, China). The supernatants were used to determine the concentrations of IL- $1 \beta$, TNF- $\alpha$, and IL-10 using a commercial mice ELISA kit (Jiangsu Jingmei Biological Technology Co. Ltd, Jiangsu, China), respectively. The level of sensitivity of each kit was $0.1 \mathrm{pg} / \mathrm{mL}$ for each cytokine (18).

\section{Reverse Transcription-Quantitative Polymerase Chain Reaction (RT-qPCR)}

Samples of liver tissues $(30 \mathrm{mg} / \mathrm{mouse})$ were snap-frozen with liquid $\mathrm{N}_{2}$ and using a ceramic mortar, tissues were grinded into powder. Following the manufacturer's instructions. total RNA from each sample was extracted using an Animal Total RNA Isolation Kit (Sagon Biotech, Shanghai, China). After validating the isolated RNA concentration and purity using the NanoDrop One system (Thermo Fisher Scientific, Waltham, MA; OD260/ $280 \approx 1.9-2.0$ ), cDNA was prepared form triplicate aliquots (each $1 \mu \mathrm{g})$ using a PrimeScrip RT reagent kit (Takara, Tokyo Japan). Thereafter, qRT-PCR was performed using a SYBR Premix ExTaq (Takara) and a CFX96 thermal cycler (BioRad, Hercules, CA). The PCR conditions were shown as follows: $95^{\circ} \mathrm{C}$ for $5 \mathrm{~min}$, followed by 40 cycles of $95^{\circ} \mathrm{C}, 15 \mathrm{~s}$ for denaturation, $60^{\circ} \mathrm{C}, 60 \mathrm{~s}$ for annealing at and $70^{\circ} \mathrm{C}, 25 \mathrm{~s}$ for extension. Each qRT-PCR reaction was performed with volumes of $10 \mu \mathrm{L}$ containing $5 \mu \mathrm{L}$ TB Green TM Premix (Takara), $1 \mu \mathrm{L}$ forward and reverse primers, $1 \mu \mathrm{L} c D N A$, and 2 $\mu \mathrm{L}$ DNase/RNase-Free Deionized Water (Tiangen, Beijing, China). The primers used to analyze the genes of interest were designed from NCBI genBank and are shown in Table 2. Relative gene expression in each sample was normalized to an internal control ( $\beta$-actin); data analysis was performed using the $2-{ }^{\Delta \Delta \mathrm{Ct}}$ method. All samples were evaluated in triplicate.

\section{Statistical Analysis}

Statistical analysis of the data collected (from various independent experiments) was performed using GraphPad

TABLE 2 | Primers used for the real-time PCR analysis.

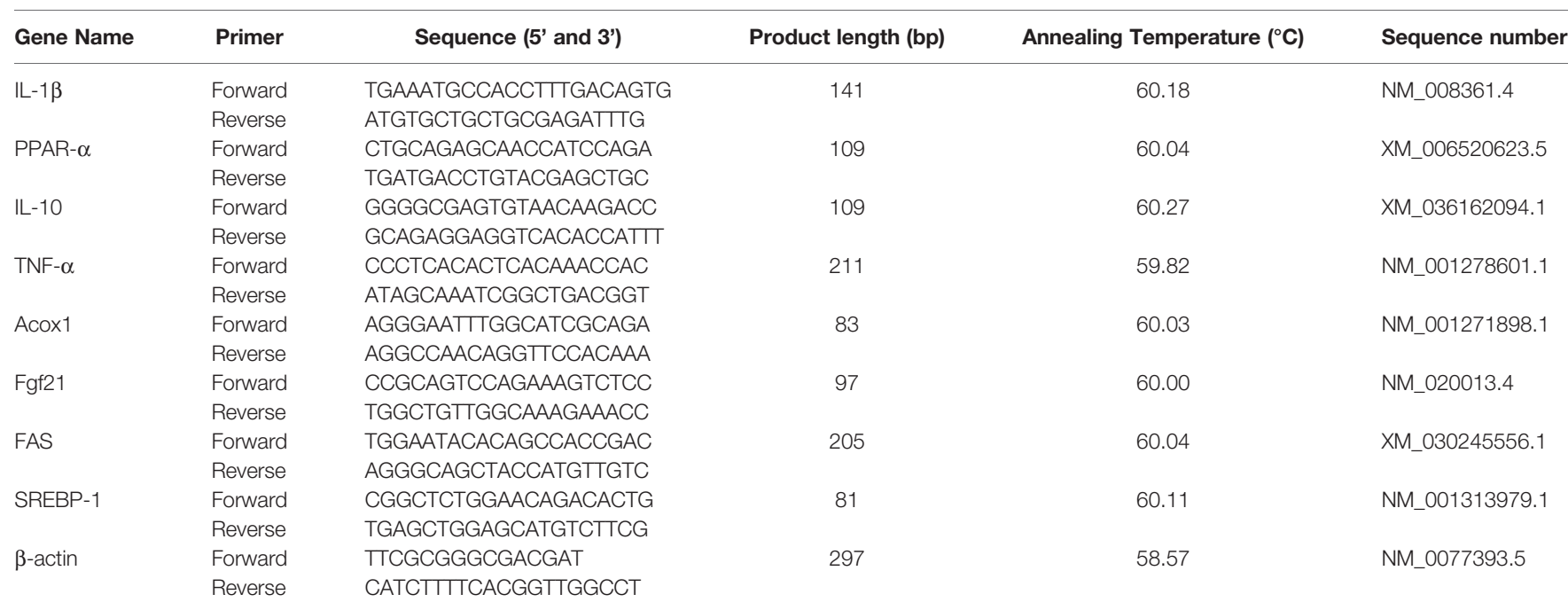


Prism 5.04 software (GraphPad Software, Inc., La Jolla, CA, USA) and SPSS 20 Statistical Analysis Software (SPSS Inc., Chicago, IL, USA). The Shapiro-Wilk Test was used to test the normality of the data. All experimental results are presented as mean $\pm \mathrm{SD}$, and statistical significance were determined by oneway analysis of variance (ANOVA) followed by the Tukey's test. The values were significantly different at $\mathrm{P}<0.05$.

\section{RESULTS}

\section{Effects of B. toyo SAU-20 on Growth Performance in HFD/STZ-Induced T2DM Mice}

From the results, during the experimental trail we observed a significant increase in feed and water intake in the DBG group compared to the control (C) and B. toyo SAU-20 groups (Table 3, $\mathrm{P}<0.05$ ), typical of T2DM. However, the level of feed and water intake in the $B$. toyo SAU-20 was higher compared to the control group. $(\mathrm{P}<0.05)$. Furthermore, we observed a decrease in the weight gain in the DBG group after the 35-days compared to the control (C) and B. toyo SAU-20 groups even though feed intake was high in the DBG group $(\mathrm{P}<0.05)$. No difference existed in weight gain between the control $(C)$ and $B$. toyo SAU-20 groups. Moreover, we observed a significant decrease in liver, kidney and spleen indices in the diabetes group compared to the control and $B$. toyo SAU-20 groups $(\mathrm{P}<0.05)$. We also observed an increase in weight of abdominal fat in the diabetic mice compared to the control and probiotic groups.

\section{Effects of B. toyo SAU-20 on Blood Glucose Increase and Oral Glucose Tolerance Test (OGTT) in HFD/STZ- Induced T2DM Mice}

As showed in Figure 1A, the fasting blood glucose level of the DBG group after the 35-day administration period was higher as compared to the control (C) and B. toyo SAU-20 groups $(\mathrm{P}<0.05)$. However, there was no difference in fasting blood glucose between the control (C) and B. toyo SAU-20 groups. Furthermore, we observed a larger glucose area under the curve (AUC) in DBG mice as compared to the control (C) (Figure 1B, $\mathrm{P}<0.05)$. The glucose AUC was significantly lowered following oral administration of B. toyo SAU-20 to mice compared to the DBG $(\mathrm{P}<0.05)$, with no significant difference compared to control (C) group $(\mathrm{P}>0.05)$.

\section{Effects of B. toyo SAU-20 on Biochemical Parameters in HFD/STZ Induced T2DM Mice}

As shown in Table 4, the levels of AST, ALT, fructosamine, and HOMA-IR in the diabetic (DBG) group were significantly higher compared to those of the control (C) group whereas the insulin level was lower in the diabetic group $(\mathrm{P}<0.05)$. However, the oral administering of $B$. toyo SAU-20 significantly decreased the levels of biochemical parameters (AST, ALT, fructosamine, and HOMA-IR) and increase the levels of insulin compared to group DG mice $(\mathrm{P}<0.05)$. However, the frutosamine and HOMA-IR levels of the B. toyo SAU-20 group was significantly higher than the control $(\mathrm{C})$ group.

Liver glycogen content was lower in DBG group compared to the control $(\mathrm{C})$ and $B$. toyo SAU-20 groups $(\mathrm{P}>0.05)$.

\section{Effects of B. toyo SAU-20 on Lipid Profile in HFD/STZ Induced T2DM Mice}

The effects of $B$. toyo SAU-20 on the lipid profile are shown in Table 4. TC, TG and LDL-C levels in Diabetic group (DBG) were higher compared to control (C) group in both serum and liver $(\mathrm{P}<0.05)$. However, these parameters were significantly attenuated in the $B$. toyo SAU-20 group $(\mathrm{P}<0.05)$. Moreover, the TC (serum and liver), TG (Liver), LDL-C (serum) levels in the $B$. toyo SAU-20 group were increased as compared to the control $(C)$ group $(\mathrm{P}<0.05)$. There were no significant differences in TG (serum) and LDL-C (liver) levels between B. toyo SAU-20 group and control (C) group. The HDL-C levels in the Diabetic group (DBG) were decreased as compared to the B. toyo SAU-20 group and control (C) group $(\mathrm{P}<0.05)$, however, there were no significant differences in HDL-C levels between $B$. toyo SAU-20 group and control (C) group.

TABLE 3 | Effects of B. toyo SAU-20 on growth performance in HFD/STZ-induced T2DM mice.

\begin{tabular}{|c|c|c|c|}
\hline \multirow[t]{2}{*}{ Growth parameters } & \multicolumn{3}{|c|}{ Groups } \\
\hline & C & DBG & Bacillus toyo. SAU-2C \\
\hline Daily feed intake (g/day) & $10.03 \pm 0.80^{b}$ & $22.72 \pm 2.21^{\mathrm{a}}$ & $13.87 \pm 0.80^{b}$ \\
\hline Daily water intake (ml/day) & $7.49 \pm 0.88^{c}$ & $14.61 \pm 0.44^{a}$ & $11.35 \pm 2.43^{b}$ \\
\hline Average body weight $(\mathrm{g})$ & $47.69 \pm 0.79^{a}$ & $37.33 \pm 2.02^{c}$ & $43.96 \pm 1.44^{b}$ \\
\hline Liver index (\%) & $2.24 \pm 0.31^{a}$ & $1.42 \pm 0.31^{b}$ & $2.19 \pm 0.32^{a}$ \\
\hline Kidney index (\%) & $0.26 \pm 0.03^{a}$ & $0.18 \pm 0.04^{b}$ & $0.20 \pm 0.05^{b}$ \\
\hline spleen index (\%) & $0.49 \pm 0.03^{a}$ & $0.35 \pm 0.04^{b}$ & $0.50 \pm 0.05^{\mathrm{a}}$ \\
\hline Abdominal fat index (\%) & $0.03 \pm 0.01^{c}$ & $0.16 \pm 0.05^{a}$ & $0.11 \pm 0.03^{b}$ \\
\hline
\end{tabular}

The table is represented as means value \pm standard deviation (SD). values in the same row with different superscripts a-c are statistically different ( $n=8, P<0.05$ ). $C$, normal group, blank control mice without HFD/STZ treatment; DBG, T2DM model mice with HFD/STZ treatment; DBG + B. toyo SAU-20, mice with HFD/STZ treatment and oral gavage administration of B. toyo SAU-20.

Organ indices, Organ weight/Body weight $X 100 \%$. 

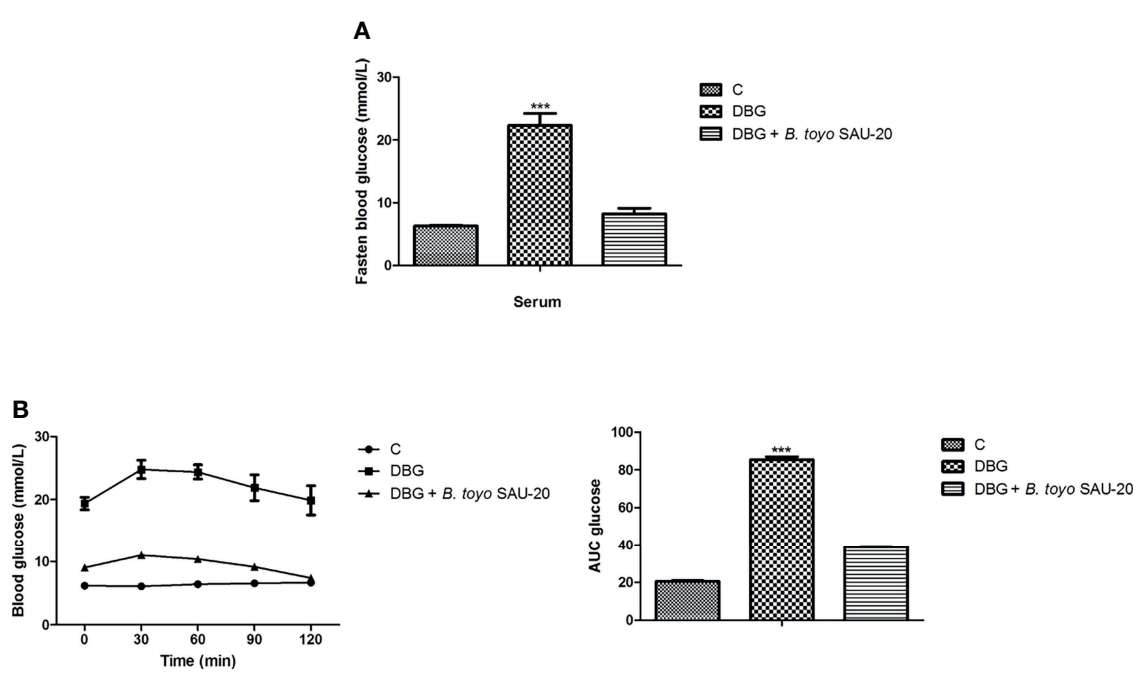

FIGURE 1 | Effects of B. toyo SAU-20 on blood glucose increase in HFD/STZ-induced T2DM mice. (A) Fasting blood glucose (mmo/L) (B) Oral glucose tolerance test $(\mathrm{mmol} / \mathrm{L})$. Values were showed by mean $\pm \mathrm{Sd}$. ${ }^{\star \star \star} P \mathrm{P}<0.05 \mathrm{DBG}$ compared with the Control (C) and DBG $+B$. toyo SAU-20 groups $(n=6)$.

\section{Effects of B. toyo SAU-20 on Antioxidant Parameters in HFD/STZ Induced T2DM Mice}

The deviations in oxidative stress status of the mice livers are presented in Table 4. As compared to control (C) group, the oxidative stress component MDA increased in the DBG whereas the antioxidative stress components (SOD and GSH) in the DBG group was greatly decreased $(\mathrm{P}<0.05)$. However, the oral administration of $B$. toyo SAU-20 reverted these effects by increasing the levels of antioxidative stress components (SOD and GSH) and reducing the levels of oxidative stress component MDA $(\mathrm{P}<0.05)$. Moreover, the levels of antioxidative

TABLE 4 | Effects of B. toyonensis SAU-20 on biochemical parameters in HFD/STZ-induced T2DM mice.

\begin{tabular}{|c|c|c|c|c|}
\hline \multirow[t]{2}{*}{ Tissue } & \multirow[t]{2}{*}{ Biochemical parameters } & \multicolumn{3}{|c|}{ Groups } \\
\hline & & C & DBG & $\mathrm{DBG}+$ B. toyo SAU-20 \\
\hline \multirow[t]{5}{*}{ Blood } & AST (U/L) & $130.67 \pm 2.08^{c}$ & $253.0 \pm 14.93^{a}$ & $201.33 \pm 5.43^{b}$ \\
\hline & $\mathrm{ALT}(\mathrm{U} / \mathrm{L})$ & $28.67 \pm 1.53^{\mathrm{C}}$ & $114.67 \pm 3.51^{\mathrm{a}}$ & $65.33 \pm 4.51^{b}$ \\
\hline & Fructosamine $(\mu \mathrm{mol} / \mathrm{L})$ & $136.33 \pm 3.51^{b}$ & $445.87 \pm 92.62^{\mathrm{a}}$ & $262.97 \pm 7.42^{\mathrm{b}}$ \\
\hline & Blood insulin (mU/L) & $13.52 \pm 0.39^{a}$ & $9.01 \pm 0.73^{b}$ & $13.23 \pm 2.12^{a}$ \\
\hline & HOMA-IR & $3.80 \pm 0.16^{b}$ & $8.91 \pm 0.20^{\mathrm{a}}$ & $4.82 \pm 0.80^{b}$ \\
\hline Liver & $\begin{array}{l}\text { Liver glycogen } \\
\text { (mg/g protein) }\end{array}$ & $4.80 \pm 0.30^{a}$ & $1.57 \pm 0.45^{b}$ & $4.10 \pm 0.17^{a}$ \\
\hline \multicolumn{5}{|c|}{ Lipid profiles } \\
\hline \multirow[t]{4}{*}{ Blood } & $\mathrm{TC}(\mathrm{mmol} / \mathrm{L})$ & $2.61 \pm 0.56^{c}$ & $5.12 \pm 0.21^{a}$ & $3.67 \pm 0.21^{b}$ \\
\hline & $\mathrm{TG}(\mathrm{mmol} / \mathrm{L})$ & $1.40 \pm 0.08^{b}$ & $2.74 \pm 0.07^{a}$ & $1.56 \pm 0.14^{b}$ \\
\hline & LDL-C (mmol/L) & $2.41 \pm 0.17^{\mathrm{c}}$ & $4.37 \pm 0.04^{a}$ & $3.07 \pm 0.06^{b}$ \\
\hline & HDL-C (mmol/L) & $0.23 \pm 0.04^{a}$ & $0.10 \pm 0.03^{b}$ & $0.20 \pm 0.04^{a}$ \\
\hline \multirow[t]{4}{*}{ Liver } & $\mathrm{TC}(\mathrm{mmol} / \mathrm{L})$ & $2.13 \pm 0.18^{c}$ & $4.71 \pm 0.16^{a}$ & $3.07 \pm 0.04^{b}$ \\
\hline & $\mathrm{TG}(\mathrm{mmol} / \mathrm{L})$ & $0.92 \pm 0.07^{c}$ & $2.06 \pm 0.07^{a}$ & $1.12 \pm 0.06^{b}$ \\
\hline & LDL-C (mmol/L) & $1.27 \pm 0.06^{b}$ & $3.08 \pm 0.06^{a}$ & $1.46 \pm 0.11^{b}$ \\
\hline & HDL-C (mmol/L) & $0.17 \pm 0.02^{a}$ & $0.02 \pm 0.01^{b}$ & $0.14 \pm 0.03^{a}$ \\
\hline \multicolumn{5}{|c|}{ Oxidative stress } \\
\hline \multirow[t]{3}{*}{ Blood } & MDA (nmol/mL) & $0.54 \pm 0.04^{b}$ & $1.82 \pm 0.12^{\mathrm{a}}$ & $0.67 \pm 0.14^{b}$ \\
\hline & $\mathrm{SOD}(\mathrm{ng} / \mathrm{mL})$ & $9.45 \pm 0.57^{\mathrm{a}}$ & $5.69 \pm 0.39^{b}$ & $9.10 \pm 0.75^{a}$ \\
\hline & GSH (ng/mL) & $1.42 \pm 0.15^{\mathrm{a}}$ & $0.49 \pm 0.07^{c}$ & $0.90 \pm 0.06^{b}$ \\
\hline \multirow[t]{3}{*}{ Liver } & MDA (nmol/mL) & $0.59 \pm 0.03^{b}$ & $0.88 \pm 0.07^{a}$ & $0.60 \pm 0.02^{b}$ \\
\hline & $\mathrm{SOD}(\mathrm{ng} / \mathrm{mL})$ & $7.55 \pm 0.41^{a}$ & $4.18 \pm 0.37^{b}$ & $7.83 \pm 0.89^{a}$ \\
\hline & GSH (ng/mL) & $1.05 \pm 0.08^{a}$ & $0.33 \pm 0.03^{c}$ & $0.84 \pm 0.16^{b}$ \\
\hline
\end{tabular}

Data represent mean $\pm S D(n=5)$, values in the same row with different superscript letters are significantly different $(P<0.05)$. C, normal group, blank control mice without HFD/STZ treatment; DBG, T2DM model mice with HFD/STZ treatment; DBG + B. toyo SAU-20, mice with HFD/STZ treatment and oral gavage administration of B. toyonensis SAU-20.

AST, Aspartate Aminotransferase; ALT, Alanine Aminotransferase; TC, total cholesterol; TG, total triglyceride; LDL-C, LDL-cholesterol; HDL-C, HDL-cholesterol; GSH, glutathione; GSH$P X$, glutathione peroxide; CAT, catalase; SOD, superoxide dismutase; MDA, malondialdehyde. 
components (GSH) in the $B$. toyo SAU-20 group were significantly lower compared to the control group $(\mathrm{P}<0.05)$.

\section{Effects of B. toyo SAU-20 on Hepatic Steatosis in HFD/STZ Induced T2DM Mice}

As represented in Figure $\mathbf{2 A}$, we observed hepatomegaly, vesicular degeneration and prominent diffuse macrovesicular steatosis in livers of the diabetic mice (DBG) compared to the control group that showed normal histological appearance of the liver (C). However, oral administration of B. toyo SAU-20 reversed the accumulation of fats induced by $\mathrm{HFD} / \mathrm{STZ}$ treatment (Figure 2B).

\section{Effects of B. toyo SAU-20 on Relative mRNA and Protein (ELISA) Expression of Genes Related Inflammation in Liver Tissues of HFD/STZ Induced T2DM Mice}

As shown in Figure 3. The mRNA expression levels of genes of pro-inflammatory cytokines (IL-1 $\beta$ and TNF- $\alpha$ ) were significantly elevated whereas anti-inflammatory cytokine (IL$10)$ were reduced in the diabetic (DBG) group compared to the control (Figures 3A-C, $\mathbf{P}<0.05$ ). However, B. toyo SAU-20 reduced the expression levels of pro-inflammatory cytokines and increased the expression of anti-inflammatory cytokines compared to the DBG group $(\mathrm{p}<0.05)$ but the expression levels of both pro- and anti- inflammatory cytokines were different to the control $(\mathrm{P}<0.05)$. Similarly, the protein (ELISA) expression levels of pro-inflammatory cytokines were higher whereas the anti-inflammation cytokines were lower in the DBG group compared to the control and the $B$. toyo SAU-20 groups (Figures 3D-F). However, the protein level of IL-10 in the SAU-20 group was significantly lower that the control.

\section{Effects of B. toyo SAU-20 on Relative mRNA Expression of Genes Related to Lipogenesis and Fatty Acid Oxidation in Liver Tissues of HFD/STZ Induced T2DM Mice}

As shown in Figure 4. The mRNA expression levels of peroxisome proliferator activated receptor (PPAR $\alpha$ ) was decreased in the T2DM mice group compared to the control and the $B$. toyo SAU-20 group (Figure $4 \mathbf{A}, \mathrm{P}<0.05$ ). However, the levels of PPAR $\alpha$ in the $B$. toyo SAU-20 group was significantly lower than the control group. In addition, the expression levels of genes related to lipogenesis, fatty acid lipogenesis synthase (FAS) and sterol regulatory element binding protein-1 (SREBP-1) were upregulated in the Diabetic (DBG) group compared to the control and the feeding of $B$. toyo SAU-20 downregulated these genes (Figures $4 \mathbf{B}, \mathbf{C}, \mathrm{P}<0.05$ ), but the expression levels were higher compared to the control group $(\mathrm{P}<0.05)$. Furthermore, genes related to fatty acid oxidation, Acox1 and Fgf21 were downregulated in the DBG group compared to the control group but the oral administration of $B$. toyo SAU-20 reverted this effect by increasing the expression levels of Acox1 and Fgf21, however, was significantly lower than the control group (Figure $4 \mathrm{D}, \mathrm{P}<0.05$ ).

\section{DISCUSSION}

Numerous studies have reported on novel therapeutic agents for treating treatment of type 2 diabetes (19-23). One of the strategies that have been developed and in use is the oral administration of probiotics to modulate intestinal microbiota, which has been associated with the treatment or delay of the

A
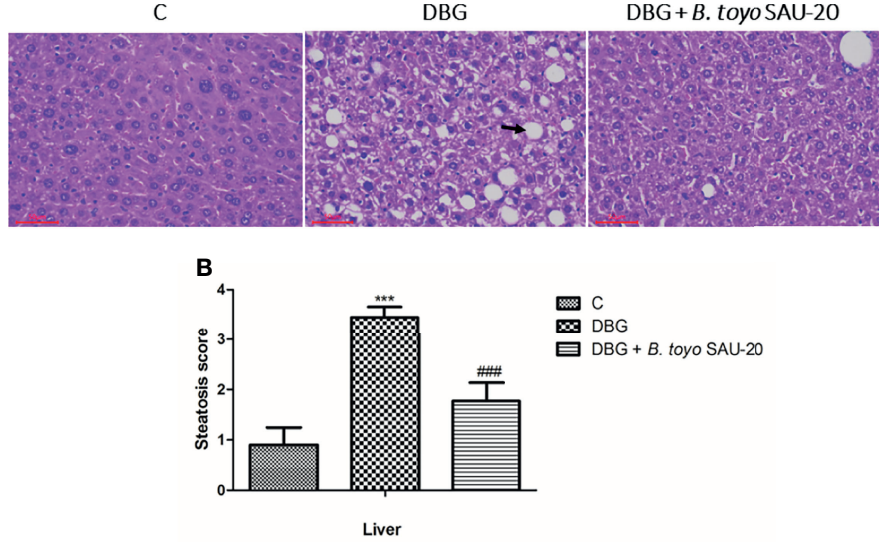

FIGURE 2 | Effects of B. toyo SAU-20 on hepatic steatosis in HFD/STZ-induced T2DM mice. (A) shows the histological results of mice liver observed at magnification $200 \times$. C- Liver section from control mice showed normal appearance of liver cells; DBG-Liver section from HFD.STZ mice showed marked fatty infiltration of hepatocytes with macro- or micro-vesicular steatosis (black arrow); DGB + B. toyo SAU-20- Liver section from B. toyo SAU-20 mice showed mild fatty infiltration of hepatocytes. The result shown here was from one representative experiment of four different samples with similar results $(n=5)$. (B) Hepatic steatosis score. Values were showed by mean $\pm \mathrm{Sd} .{ }^{* \star *} \mathrm{P}<0.05 \mathrm{DBG}$ compared with the Control (C) and DBG + B. toyo SAU-20 groups. ${ }^{\# \# \#}<0.05$ DGB + B. toyo SAU-20 compared with the Control (C) group. 

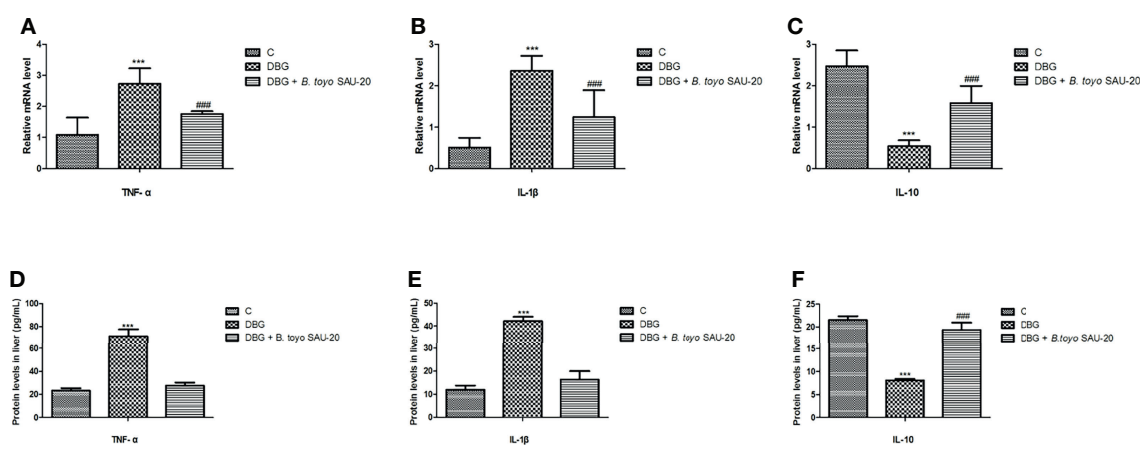

FIGURE 3 | Effects of B. toyo SAU-20 on relative mRNA and protein (ELISA) expressions of pro- and anti-inflammation related cytokines in HFD/STZ-induced T2DM mice. (A-C) relative mRNA levels of pro- and anti-inflammatory cytokines (D-F) Protein levels of pro- and anti-inflammatory cytokines Values were showed as mean \pm Sd. ${ }^{\star \star \star} \mathrm{P}<0.05$ DBG compared with the Control (C) and DBG + B. toyo SAU-20 groups. ${ }^{\# \# \# ~} \mathrm{P}<0.05 \mathrm{DGB}+\mathrm{B}$. toyo SAU-20 compared with the Control (C) group (n=8).

onset diabetes $(24,25)$. Lactobacillus and Bifidobacterium species have been reported to improve glucose tolerance, glucoseinduced insulin secretion, stabilized inflammatory activities, and decrease the frequency of diabetes (26). In this study we tested the effect of $B$. toyo SAU-20 a novel probiotic bacteria isolated by our lab on a well-established diabetic mice model, which was induced by HFD feeding along with intraperitoneal STZ injection. The success of the T2DM mice model were measured by observing the usual phenotypes of T2DM such as weight loss, increased food and water consumption, hyperglycemia, and insulin resistance (27). Hepatic steatosis is a common disorder of T2DM $(28,29)$. Inequalities in hepatic lipid metabolism results in the accumulation of hepatic triglycerides and insulin resistance, which finally cause hepatic steatosis (30). Our results revealed that the body, liver, and spleen weights of the diabetic mice were lower than those of control group. These results were similar with previous reports by Zafar and Naeem-Ul-Hassan Naqvi, (31). Moreover, the administration of SAU-20 maintained the body weights and prevented weight loss by T2DM induced diabetes.

Impaired glucose tolerance is a key parameter for type 2 diabetes (32). Our results from fasten glucose and OGTT indicated that blood glucose levels in the diabetic group were elevated compared to the control. However, the oral administration of $B$. toyo SAU-20 reduced the glucose levels in the blood. These results demonstrated that oral administration of B. toyo SAU-20 may improve glucose tolerance and prevent the development of hyperglycemia in type 2 diabetic mice.

Various studies have reported that diabetes causes a rise in the AST and ALT levels $(33,34)$. Similarly, in this study we observed an increase in the levels of liver injury markers in the blood, however, the oral administration of $B$. toyo SAU-20 reduced the levels of these liver inflammation and damage markers. This result was similar to the study by Hsieh et al. (35) who reported that Lactobacillus salivarius AP-32 and Lactobacillus reuteri GL104 (probiotic) could reduce the levels of AST and ALT in type 2
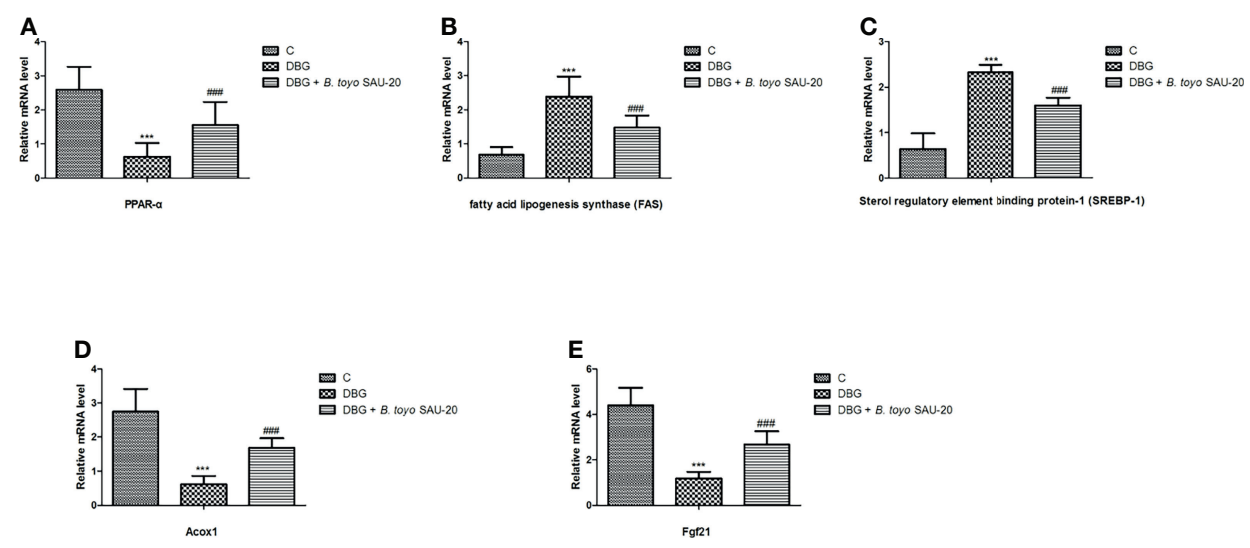

FIGURE 4 | Effects of B. toyo SAU-20 on relative mRNA expressions of genes related to lipogenesis and fatty acid oxidation in liver tissues of HFD/STZ-induced T2DM mice. (A-C) Relative mRNA levels of genes responsible for lipogenesis. (D, E) Relative mRNA levels of genes responsible for fatty acid oxidation. Values were showed by mean \pm Sd. ${ }^{* \star *} \mathrm{P}<0.05 \mathrm{DBG}$ compared with the Control (C) and DBG + B. toyo SAU-20 groups. \#\#\#P $<0.05$ DGB + B. toyo SAU-20 compared with the Control $(\mathrm{C})$ group $(\mathrm{n}=8)$. 
diabetes patients. Furthermore, we also observed that, the level of liver glycogen was much higher in the $B$. toyo SAU-20 group compared to the diabetic group, indicating that B. toyo SAU-20 could reduce blood glucose by excess glucose to glycogen in the liver. This result was similar with previous studies by Tao et al. (36).

Insulin is the major life-saving prescription for type 1 and type 2 Diabetes Mellites (37). Insulin plays active roles in growth performance, blood glucose and lipids maintenance and wound healing (38-41).

Insulin resistance was significantly higher whereas insulin levels were reduced in the diabetic mice compared to control group. However, B. toyo SAU-20 consumption increased the insulin levels and decreased the value of HOMA-IR index in HFD/STZ mice. These results indicated that B. toyo SAU-20 has the potential to increase production of insulin in HFD/STZ mice, thereby improving growth performance, insulin resistance, glucose tolerance and lipid profiles which was evident in our work.

The major pathogenesis of insulin resistance is the buildup of visceral fat which promotes increase blood pressure, dyslipidemia, and dysregulation of glucose metabolism (42). Amongst the target tissues of insulin, liver is the principal regulator of lipid metabolism via regulating lipogenesis (43). From our study, we observed the oral administration of $B$. toyo SAU-20 decreased the levels key constituents of metabolic syndrome, including serum glucose, insulin resistance, LDL, TG, and cholesterol, enhanced by HFD/STZ in both the serum and liver. Therefore, we concluded that oral administration of $B$. toyo SAU-20 could improve hepatic steatosis by reducing the production lipids in both the serum and hepatic tissues.

Oxidative stress is associated with hepatic steatosis and T2DM (44). Hyperglycemia and dyslipidemia could increase the production of reactive oxygen species (ROS) which may cause damage to living cells and specific receptors of the cell membrane and then finally cause injury to the organs such as islets of Langerhans and liver $(45,46)$. Several probiotics have been reported to show effective antioxidants activities (47). In this study, feeding of B. toyo SAU-20 significantly increased the antioxidant activities and decreased oxidative stress activity (MDA) in TD2M mice. These results indicated that probiotic strain $B$. toyo SAU-20 can reduce the activity of ROS, thus improving hepatic steatosis, insulin resistance, and preventing the damage to organs such as the pancreas, the liver, and the kidney.

Systemic and subclinical inflammation has been reported to play a vital role in Type 2 diabetes mellitus $(48,49)$. Our results showed that the expressions of pro inflammatory cytokines (IL$1 \beta$ and TNF- $\alpha$ ) in the liver was elevated while the level of anti-inflammatory (IL-10) was reduced in the diabetic group compared to the control group, however, the oral administration of B. toyo SAU-20 reverted these effects. This result was in consistent with the study by Liu et al. (50) who showed that Lactobacillus rhamnosus GG culture supernatant (LGGs) could reduce liver inflammation and injury in High-fat/high- fructose diet plus intermittent hypoxia exposure-induced metabolic dysfunction.

To reveal the possible mechanisms of B. toyo SAU-20 in suppression of hepatic lipid accumulation in TD2M mice, we evaluated the liver mRNA levels of genes involved in lipogenesis. Srebp-1c is a well-known transcription factor that regulates hepatic fatty acid and triglyceride biosynthesis by upregulating the expression of key genes, such as FAS (51). Fatty acid synthase (FAS) is a multifunctional enzyme involved in the production of fatty acids for lipid biosynthesis and is overexpressed in multiple diseases like cancer, viral, nonalcoholic fatty liver disease, and metabolic disorders (52). HFD/STZ treatment has been reported to elevates the expression of hepatic Srebp-1c, and FAS in rats (53). Our study showed that HFD/STZ treatment remarkably induced the expression of lipogenic genes (srebp-1c and FAS) in mice liver. However, the oral administration B. toyo SAU-20 counteracted the increase in hepatic lipogenic genes in HFD/STZ mice. This result was similar to the work of Hsieh et al. (3) who reported that Lactobacillus reuteri GMNL-263 could downregulate genes related to lipogenesis in liver of rats fed high fructose diet. These results suggested that B. toyo SAU-20 consumption suppressed hepatic lipid accumulation in TD2M mice.

Hepatic fatty acid oxidation is associated with fatty acid catabolism along with diabetic pathogenesis $(54,55)$. The peroxisome proliferator-activated receptors (PPARs) control the expression of key genes related to metabolic diseases including obesity, dyslipidemia and diabetes (56). PPAR- $\alpha$ is mainly expressed in the liver and is associated with maintaining lipid homeostasis (57). It promotes fatty acid $\beta$-oxidation by modulating the respective genes (58) as well as improve glucose metabolism in T2DM by increasing insulin sensitivity and reducing hyperglycemia and hyperinsulinemia (59). TG and low-density lipoprotein metabolism is reduced by PPAR- $\alpha$ activation (60). while the knockout reduce the expression of genes involved in fatty acid oxidation in the liver (6). Acox1 and Fgf21 are genes responsible for encoding the fatty acid oxidation enzymes and are regulated by PPAR- $\alpha$ (6). We found that $B$. toyo SAU-20 upregulated the expression of PPAR- $\alpha$ and its target genes involved in fatty acid oxidation (Acox1 and Fgf21) in the liver of diabetic mice. Therefore, one alternative therapeutic approach for diabetes could be the amelioration of hepatic steatosis by activating PPAR- $\alpha$ pathway. However, this requires further research.

In conclusion, this study demonstrates that B. toyo SAU-20 improved lipid profiles and attenuated hepatic steatosis in HFD/ STZ-induced diabetic mice. The possible mechanism for these effects might be associated to $B$. toyo SAU-20 ability to decrease insulin resistance and oxidative stress, improve lipid profiles, downregulate genes responsible for lipogenesis, and upregulating genes responsible for fatty acid oxidation. These results support the use of B. toyo SAU-20 for the primary treatment of T2DM and other fat related metabolic disorders, however, there still the need for further studies to validate its use through various clinical trials. 


\section{DATA AVAILABILITY STATEMENT}

The original contributions presented in the study are included in the article/supplementary material. Further inquiries can be directed to the corresponding author.

\section{ETHICS STATEMENT}

The animal study was reviewed and approved by Institutional Animal Care and Use Committee of Sichuan Agricultural University, Sichuan, China, under the permit number DKY-B2019603005.

\section{AUTHOR CONTRIBUTIONS}

ZR, SO, LX, JWe, ZC, and JWa: Conceptualization, Methodology, Software. ZR, SO, LX, JWe, JWa, ZC, and XN:

\section{REFERENCES}

1. Khan MAB, Hashim MJ, King JK, Govender RD, Mustafa H, Al Kaabi J. Epidemiology of Type 2 Diabetes - Global Burden of Disease and Forecasted Trends. J Epidemiol Glob Health (2020) 10(1):107-11. doi: 10.2991/ jegh.k.191028.001

2. Shaw JE, Sicree RA, Zimmet PZ. Global Estimates of the Prevalence of Diabetes for 2010 and 2030. Diabetes Res Clin Pract (2010) 87(1):4-14. doi: 10.1016/j.diabres.2009.10.007

3. Hsieh FC, Lee CL, Chai CY, Chen WT, Lu YC, Wu CS. Oral Administration of Lactobacillus Reuteri GMNL-263 Improves Insulin Resistance and Ameliorates Hepatic Steatosis in High Fructose-Fed Rats. Nutr Metab (Lond) (2013) 17:35. doi: 10.1186/1743-7075-10-35

4. Loomba R, Sanyal AJ. The Global NAFLD Epidemic. Nat Rev Gastroenterol Hepatol (2013) 10(11):686-90. doi: 10.1038/nrgastro.2013.171

5. Asrih M, Jornayvaz FR. Metabolic Syndrome and Nonalcoholic Fatty Liver Disease: Is Insulin Resistance the Link? Mol Cell Endocrinol (2015) $418 \mathrm{Pt}$ 1:55-65. doi: 10.1016/j.mce.2015.02.018

6. Zhang C, Deng J, Liu D, Tuo X, Xiao L, Lai B, et al. Nuciferine Ameliorates Hepatic Steatosis in High-Fat Diet/Streptozocin-Induced Diabetic Mice Through a PPAR $\alpha /$ PPAR $\gamma$ Coactivator- $1 \alpha$ Pathway. Br J Pharmacol (2018) 175(22):4218-28. doi: 10.1111/bph.14482

7. Bril F, Cusi K. Management of Nonalcoholic Fatty Liver Disease in Patients With Type 2 Diabetes: A Call to Action. Diabetes Care (2017) 40(3):419-30. doi: $10.2337 / \mathrm{dc} 16-1787$

8. Okyere SK, Mo Q, Pei G, Ren Z, Deng J, Hu Y. Euptox A Induces G0 /GI Arrest and Apoptosis of Hepatocyte via ROS, Mitochondrial Dysfunction and Caspases-Dependent Pathways In Vivo. J. Toxicol Sci (2020) 45(11):661-71. doi: 10.2131 /jts.45.661

9. Okyere SK, Wen J, Cui Y, Xie L, Gao P, Wang J, et al. Toxic Mechanisms and Pharmacological Properties of Euptox A, a Toxic Monomer From A. Adenophora. Fitoterapia (2021) 155:105032. doi: 10.1016/j.fitote.2021.105032

10. Ren Z, Okyere SK, Wen J, Xie L, Cui Y, Wang S, et al. An Overview: The Toxicity of Ageratina Adenophora on Animals and Its Possible Interventions. Int J Mol Sci (2021) 22(21):11581. doi: 10.3390/ijms222111581

11. Okyere SK, Wen J, Cui Y, Xie L, Gao P, Zhang M, et al. Bacillus Toyonensis SAU-19 and SAU-20 Isolated From Ageratina Adenophora Alleviates the Intestinal Structure and Integrity Damage Associated With Gut Dysbiosis in Mice Fed High Fat Mice. Front Microbiol (2021). doi: 10.3389/ fmicb.2022.820236

12. Nath S, Ghosh SK, Choudhury Y. A Murine Model of Type 2 Diabetes Mellitus Developed Using a Combination of High Fat Diet and Multiple Low
Data collection, Writing, Original draft preparation. SO, JWe, ZC, and JD: Validation, Investigation. ZR, SO, JWe, XN, JD and YH: Review editing, Funding, Supervision. All authors have read and agreed to the published version of the manuscript.

\section{FUNDING}

This research was supported by Sichuan Province Science and Technology Support Program (Grant No. 2020YFS0337).

\section{ACKNOWLEDGMENTS}

I would like to thank all authors for their hard work in making this paper publishable. I also extend my sincere gratitude to the teaching staff of the College of Veterinary Medicine, Sichuan Agricultural University, Chengdu, for their guidance and criticisms in writing this paper.

Doses of Streptozotocin Treatment Mimics the Metabolic Characteristics of Type 2 Diabetes Mellitus in Humans. J Pharmacol Toxicol Methods (2017) 84:20-30. doi: 10.1016/j.vascn.2016.10.007

13. Zeng Z, Yuan Q, Yu R, Zhang J, Ma H, Chen S. Ameliorative Effects of Probiotic Lactobacillus Paracasei NL41 on Insulin Sensitivity, Oxidative Stress, and Beta-Cell Function in a Type 2 Diabetes Mellitus Rat Model. Mol Nutr Food Res (2019) 63(22):e1900457. doi: 10.1002/mnfr.201900457

14. Yu Q, Li J, Dai C-L, Li H, Iqbal K, Liu F, et al. Anesthesia With Sevoflurane or Isoflurane Induces Severe Hypoglycemia in Neonatal Mice. PloS One (2020) 15(4):e0231090. doi: 10.1371/journal.pone.0231090

15. Matthews DR, Hosker JP, Rudenski AS, Naylor BA, Treacher DF, Turner RC Homeostasis Model Assessment: Insulin Resistance and Beta-Cell Function From Fasting Plasma Glucose and Insulin Concentrations in Man. Diabetologia (1985) 28(7):412-9. doi: 10.1007/BF00280883

16. Zheng X, Zhao MG, Jiang CH, Sheng XP, Yang HM, Liu Y, et al. Triterpenic Acids-Enriched Fraction From Cyclocarya Paliurus Attenuates Insulin Resistance and Hepatic Steatosis via PI3K/Akt/GSK3 $\beta$ Pathway. Phytomedicine (2020) 66:153130. doi: 10.1016/j.phymed.2019.153130

17. Qayyum A, Chen DM, Breiman RS, Westphalen AC, Yeh BM, Jones KD, et al. Evaluation of Diffuse Liver Steatosis by Ultrasound, Computed Tomography, and Magnetic Resonance Imaging: Which Modality Is Best? Clin Imaging (2009) 33(2):110-5. doi: 10.1016/j.clinimag.2008.06.036

18. Cui Y, Okyere SK, Gao P, Wen J, Cao S, Wang Y, et al. Ageratina Adenophora Disrupts the Intestinal Structure and Immune Barrier Integrity in Rats. Toxins (Basel) (2012) 13(9):651. doi: 10.3390/toxins13090651

19. Yamada Y, Katagiri H, Hamamoto Y, Deenadayalan S, Navarria A, Nishijima $\mathrm{K}$, et al. Dose-Response, Efficacy, and Safety of Oral Semaglutide Monotherapy in Japanese Patients With Type 2 Diabetes (PIONEER 9): A 52-Week, Phase 2/3a, Randomised, Controlled Trial. Lancet Diabetes Endocrinol (2020) 8(5):377-91. doi: 10.1016/S2213-8587(20)30075-9

20. Flory J, Lipska K. Metformin in 2019. JAMA (2019) 321(19):1926-7. doi: 10.1001/jama.2019.3805

21. Mosenzon O, Miller EM, Warren ML. Oral Semaglutide in Patients With Type 2 Diabetes and Cardiovascular Disease, Renal Impairment, or Other Comorbidities, and in Older Patients. Postgrad Med (2020) 132(sup2):37-47. doi: 10.1080/00325481.2020.1800286

22. Nanjan MJ, Mohammed M, Prashantha Kumar BR, Chandrasekar MJN. Thiazolidinediones as Antidiabetic Agents: A Critical Review. Bioorg Chem (2018) 77:548-67. doi: 10.1016/j.bioorg.2018.02.009

23. Dalsgaard NB, Gasbjerg LS, Hansen LS, Hansen NL, Stensen S, Hartmann B, et al. The Role of GLP-1 in the Postprandial Effects of Acarbose in Type 2 Diabetes. Eur J Endocrinol (2021) 184(3):383-94. doi: 10.1530/EJE-20-1121 
24. Adeshirlarijaney A, Gewirtz AT. Considering Gut Microbiota in Treatment of Type 2 Diabetes Mellitus. Gut Microbes (2020) 11(3):253-64. doi: 10.1080/ 19490976.2020.1717719

25. Okyere SK, Xie L, Wen J, Ran Y, Ren Z, Deng J, et al. Bacillus Toyonensis SAU-19 Ameliorates Hepatic Insulin Resistance in High-Fat Diet/Streptozocin-Induced Diabetic Mice. Nutrients (2021) 13(12):4512. doi: 10.3390/nu13124512

26. Salles BIM, Cioffi D, Ferreira SRG. Probiotics Supplementation and Insulin Resistance: A Systematic Review. Diabetol Metab Syndr (2020) 12(1):98. doi: 10.1186/s13098-020-00603-6

27. Li S, Huang Q, Zhang L, Qiao X, Zhang Y, Tang F, et al. Effect of CAPE-pNO2 Against Type 2 Diabetes Mellitus via the AMPK/GLUT4/ GSK3 $\beta / P P A R \alpha$ Pathway in HFD/STZ-Induced Diabetic Mice. Eur J Pharmacol (2019) 853:110. doi: 10.1016/j.ejphar.2019.03.027

28. Khandelwal R, Dassanayake AS, Conjeevaram HS, Singh SP. Non-Alcoholic Fatty Liver Disease in Diabetes: When to Refer to the Hepatologist? World J Diabetes (2021) 12(9):1479-93. doi: 10.4239/wjd.v12.i9.1479

29. Tanase DM, Gosav EM, Costea CF, Ciocoiu M, Lacatusu CM, Maranduca MA, et al. The Intricate Relationship Between Type 2 Diabetes Mellitus (T2DM), Insulin Resistance (IR), and Nonalcoholic Fatty Liver Disease (NAFLD). J Diabetes Res (2020) 2020:3920196. doi: 10.1155/2020/3920196

30. Geng Y, Faber KN, de Meijer VE, Blokzijl H, Moshage H. How Does Hepatic Lipid Accumulation Lead to Lipotoxicity in Non-Alcoholic Fatty Liver Disease? Hepatol Int (2021) 15(1):21-35. doi: 10.1007/s12072-020-10121-2

31. Zafar M, Naeem-ul-Hassan Naqvi S. Effects of STZ-Induced Diabetes on the Relative Weights of Kidney, Liver and Pancreas in Albino Rats: A Comparative Study. Int J Morphol (2010) 28:135-42. doi: 10.4067/S071795022010000100019

32. Xu D, Jiang Z, Sun Z, Wang L, Zhao G, Hassan HM, et al. Mitochondrial Dysfunction and Inhibition of Myoblast Differentiation in Mice With HighFat-Diet-Induced Pre-Diabetes. J Cell Physiol (2019) 234(5):7510-23. doi: $10.1002 /$ jcp. 27512

33. Kuchay MS, Krishan S, Mishra SK, Choudhary NS, Singh MK, Wasir JS, et al. Effect of Dulaglutide on Liver Fat in Patients With Type 2 Diabetes and NAFLD: Randomised Controlled Trial (D-LIFT Trial). Diabetologia (2020) 63 (11):2434-45. doi: 10.1007/s00125-020-05265-7

34. Zhao W, Zhang L, Zhang G, Varkaneh HK, Rahmani J, Clark C, et al. The Association of Plasma Levels of Liver Enzymes and Risk of Gestational Diabetes Mellitus: A Systematic Review and Dose-Response Meta-Analysis of Observational Studies. Acta Diabetol (2020) 57(6):635-44. doi: 10.1007/ s00592-019-01458-8

35. Hsieh PS, Ho HH, Hsieh SH, Kuo YW, Tseng HY, Kao HF, et al. Lactobacillus Salivarius AP-32 and Lactobacillus Reuteri GL-104 Decrease Glycemic Levels and Attenuate Diabetes-Mediated Liver and Kidney Injury in $\mathrm{Db} / \mathrm{Db}$ Mice. BMJ Open Diabetes Res Care (2020) 8(1):e001028. doi: 10.1136/bmjdrc-2019-001028

36. Tao YW, Gu YL, Mao XQ, Zhang L, Pei YF. Effects of Probiotics on Type II Diabetes Mellitus: A Meta-Analysis. J Transl Med (2020) 18(1):30. doi: 10.1186/s12967-020-02213-2

37. Sharma AK, Taneja G, Kumar A, Sahu M, Sharma G, Kumar A, et al. Insulin Analogs: Glimpse on Contemporary Facts and Future Prospective. Life Sci (2019) 219:90-9. doi: 10.1016/j.lfs.2019.01.011

38. Sato K, Aoki M, Kondo R, Matsushita K, Akiba Y, Kamada T. Administration of Insulin to Newly Hatched Chicks Improves Growth Performance via Impairment of MyoD Gene Expression and Enhancement of Cell Proliferation in Chicken Myoblasts. Gen Comp Endocrinol (2012) 175 (3):457-63. doi: 10.1016/j.ygcen.2011.11.043

39. Dimitriadis GD, Maratou E, Kountouri A, Board M, Lambadiari V. Regulation of Postabsorptive and Postprandial Glucose Metabolism by Insulin-Dependent and Insulin-Independent Mechanisms: An Integrative Approach. Nutrients (2021) 13(1):159.

40. Kumar M, Ojha S, Rai P, Joshi A, Kamat SS, Mallik R. Insulin Activates Intracellular Transport of Lipid Droplets to Release Triglycerides From the Liver. J Cell Biol (2019) 218(11):3697-713. doi: 10.1083/jcb.201903102

41. Oryan A, Alemzadeh E. Effects of Insulin on Wound Healing: A Review of Animal and Human Evidences. Life Sci (2017) 174:59-67. doi: 10.1016/ j.lfs.2017.02.015

42. Hardy OT, Czech MP, Corvera S. What Causes the Insulin Resistance Underlying Obesity? Curr Opin Endocrinol Diabetes Obes (2012) 19(2):817. doi: 10.1097/MED.0b013e3283514e13
43. Alves-Bezerra M, Cohen DE. Triglyceride Metabolism in the Liver. Compr Physiol (2017) 8(1):1-8. doi: 10.1002/cphy.c170012

44. Yaribeygi H, Sathyapalan T, Atkin SL, Sahebkar A. Molecular Mechanisms Linking Oxidative Stress and Diabetes Mellitus. Oxid Med Cell Longev (2020) 2020:8609213. doi: 10.1155/2020/8609213

45. Hao JS, Zhu CJ, Yan BY, Yan CY, Ling R. Stimulation of KLF14/PLK1 Pathway by Thrombin Signaling Potentiates Endothelial Dysfunction in Type 2 Diabetes Mellitus. BioMed Pharmacother (2018) 99:859-66. doi: 10.1016/ j.biopha.2018.01.151

46. Zhou S, Dai YM, Zeng XF, Chen HZ. Circadian Clock and Sirtuins in Diabetic Lung: A Mechanistic Perspective. Front Endocrinol (Lausanne) (2020) 11:173. doi: $10.3389 /$ fendo. 2020.00173

47. Feng Z, Song X, Zhao L, Zhu W. Isolation of Probiotics and Their Effects on Growth, Antioxidant and Non-Specific Immunity of Sea Cucumber Apostichopus Japonicus. Fish Shellfish Immunol (2020) 106:1087-94. doi: 10.1016/j.fsi.2020.08.049

48. Donath MY. Multiple Benefits of Targeting Inflammation in the Treatment of Type 2 Diabetes. Diabetologia (2016) 59(4):679-82. doi: 10.1007/s00125-0163873-z

49. Elimam H, Abdulla AM, Taha IM. Inflammatory Markers and Control of Type 2 Diabetes Mellitus. Diabetes Metab Syndr (2019) 13(1):800-4. doi: 10.1016/j.dsx.2018.11.061

50. Liu Q, Liu Y, Li F, Gu Z, Liu M, Shao T, et al. Probiotic Culture Supernatant Improves Metabolic Function Through FGF21-Adiponectin Pathway in Mice. J Nutr Biochem (2020) 75:108256. doi: 10.1016/j.jnutbio.2019.108256

51. Strable MS, Ntambi JM. Genetic Control of De Novo Lipogenesis: Role in DietInduced Obesity. Crit Rev Biochem Mol Biol (2010) 45(3):199-214. doi: $10.3109 / 10409231003667500$

52. Singh K, Graf B, Linden A, Sautner V, Urlaub H, Tittmann K, et al. Discovery of a Regulatory Subunit of the Yeast Fatty Acid Synthase. Cell (2020) 180 (6):1130-43.e20. doi: 10.1016/j.cell.2020.02.034

53. Bedi O, Srivastava N, Parsad D, Krishan P. Fatty Acid Synthase Inhibition Ameliorates Diabetes Induced Liver Injury in Rodent Experimental Model. Eur J Pharmacol (2021) 901:174078. doi: 10.1016/j.ejphar.2021.174078

54. Murea M, Freedman BI, Parks JS, Antinozzi PA, Elbeinm SC, Ma L. Lipotoxicity in Diabetic Nephropathy: The Potential Role of Fatty Acid Oxidation. Clin J Am Soc Nephrol (2010) 5(12):2373-9. doi: 10.2215/CJN.08160910

55. Wakil SJ, Abu-Elheiga LA. Fatty Acid Metabolism: Target for Metabolic Syndrome. J Lipid Res (2009) 50 Suppl(Suppl):S138-43. doi: 10.1194/ jlr.R800079-JLR200

56. Montaigne D, Butruille L, Staels B. PPAR Control of Metabolism and Cardiovascular Functions. Nat Rev Cardiol (2021) 18(12):809-23. doi: 10.1038/s41569-021-00569-6

57. Yang H, Xiao L, Wang N. Peroxisome Proliferator-Activated Receptor $\alpha$ Ligands and Modulators From Dietary Compounds: Types, Screening Methods and Functions. J Diabetes (2017) 9(4):341-52. doi: 10.1111/1753-0407.12506

58. AlNafea HM, Korish AA. Activation of the Peroxisome Proliferator-Activated Receptors (PPAR- $\alpha / \gamma)$ and the Fatty Acid Metabolizing Enzyme Protein CPT1A by Camel Milk Treatment Counteracts the High-Fat Diet-Induced Nonalcoholic Fatty Liver Disease. PPAR Res (2021) 2021:5558731. doi: $10.1155 / 2021 / 5558731$

59. Hong F, Pan S, Guo Y, Xu P, Zhai Y. PPARs as Nuclear Receptors for Nutrient and Energy Metabolism. Molecules (2019) 24(14):2545. doi: 10.3390/ molecules 24142545

60. Fruchart JC. Peroxisome Proliferator-Activated Receptor-Alpha (PPARalpha): At the Crossroads of Obesity, Diabetes and Cardiovascular Disease. Atherosclerosis (2009) 205(1):1-8. doi: 10.1016/j.atherosclerosis.2009.03.008

Conflict of Interest: YH is/was employed by New Ruipeng Pet Healthcare Group Co., Ltd., Shenzhen 518000, China.

The remaining authors declare that the research was conducted in the absence of any commercial or financial relationships that could be construed as a potential conflict of interest.

Publisher's Note: All claims expressed in this article are solely those of the authors and do not necessarily represent those of their affiliated organizations, or those of the publisher, the editors and the reviewers. Any product that may be evaluated in 
this article, or claim that may be made by its manufacturer, is not guaranteed or endorsed by the publisher.

Copyright $\odot 2022$ Ren, Okyere, Xie, Wen, Wang, Chen, Ni, Deng and Hu. This is an open-access article distributed under the terms of the Creative Commons Attribution
License (CC BY). The use, distribution or reproduction in other forums is permitted, provided the original author(s) and the copyright owner(s) are credited and that the original publication in this journal is cited, in accordance with accepted academic practice. No use, distribution or reproduction is permitted which does not comply with these terms. 